\title{
A Simple Model of the Mass Difference.
}

\author{
T. Mura \\ Department of Physics, Kyoto University - Kyoto \\ (Nuovo Cimento, 44 A, 306 (1966))
}

In refs. (1,3), the following important works were missed:

(') G. Barton: to be published in Phys. Rev.

(3) H. Mirazawa, Y. OI and M. Suzuki: Progr. Theor. Phys. Suppl., The commemoration issue of the thirtieth anniversary of the meson theory, 436 (1965). 